PROCEEDINGS OF THE

AMERICAN MATHEMATICAL SOCIETY

Volume 138, Number 5, May 2010, Pages 1889-1895

S 0002-9939(10)10212-3

Article electronically published on January 5, 2010

\title{
RIGIDITY OF CARNOT GROUPS RELATIVE TO MULTICONTACT STRUCTURES
}

\author{
FILIPPO DE MARI AND ALESSANDRO OTTAZZI
}

(Communicated by Mario Bonk)

\begin{abstract}
We prove a rigidity type result for stratified nilpotent Lie algebras which gives a positive answer to a special case of a conjecture formulated by M. Cowling and of another conjecture formulated by A. Korányi.
\end{abstract}

\section{INTRODUCTION}

The notion of a multicontact mapping refers to a (local) diffeomorphism of a differentiable manifold $M$ endowed with a subbundle $H M$ of the tangent bundle $T M$ whose sections satisfy a Hörmander type condition [5], and which splits as the sum $H M=\sum_{i \leq m} H_{i} M$ of smaller subbundles, each of which is preserved by the differential of the mapping. Examples of this sort arise naturally in subRiemannian geometry. The geometric setup we are referring to is best formalized by introducing the structure of a stratified nilpotent Lie algebra at the tangent level, and hence a very natural case to examine is that of a connected and simply connected nilpotent Lie group $G$ whose Lie algebra is stratified, viz. a Carnot group. Once a multicontact structure is selected, that is, a subbundle $H G \subset T G$ (to which we refer as the horizontal or contact bundle), together with a splitting $H G=$ $\sum_{i \leq m} H_{i} G$, a basic problem to address is the characterization of the possibly infinite dimensional Lie group of multicontact mappings. In particular, it is important to know whether it is finite dimensional, that is, when $G$ is rigid.

When no splitting of $H G$ is introduced and one simply looks at the mappings whose differentials preserve it $(m=1)$, rigidity is a classical notion that has been studied because of its applications to the theory of quasiconformal mappings [2, 7 ] and to the study of infinitesimal automorphisms of dynamical systems associated to graded Lie algebras [12, 15. In 9 it is shown that a large class of nonrigid examples exists. As for multicontact structures, the reader is referred to [3, 4, 6, , 8, 13, 14, 16,

Our main contribution, Theorem 4.1 gives a partial positive answer to questions raised by M. Cowling in [1] and by A. Korányi in [6]. More precisely, the former is:

Conjecture 1.1 (Cowling, [1]). Suppose that $\mathfrak{v}_{1}$ and $\mathfrak{v}_{2}$ are subalgebras of a stratified algebra $\mathfrak{g}$, such that $\mathfrak{g}_{-1}=\mathfrak{a}_{1}+\mathfrak{a}_{2}$, where $\mathfrak{a}_{i}=\mathfrak{v}_{i} \cap \mathfrak{g}_{-1}$, and for all $X$ in $\mathfrak{a}_{1}$, there exists $Y$ in $\mathfrak{a}_{2}$ such that $[X, Y] \neq 0$, and vice versa. Let $U$ be an open subset of the corresponding group $G=\exp \mathfrak{g}$, and let $\varphi: U \rightarrow G$ be a map that maps

Received by the editors March 31, 2009, and, in revised form, September 8, 2009.

2000 Mathematics Subject Classification. Primary 22E25, 53C24, 58D05.

Key words and phrases. Carnot group, contact map, prolongation, rigidity.

(C)2010 American Mathematical Society

Reverts to public domain 28 years from publication 
connected subsets of cosets of $V_{i}=\exp \mathfrak{v}_{i}$ into cosets of $V_{i}$ when $i=1$ and 2. Then $\varphi$ is automatically smooth, and the set of these maps is finite dimensional.

In this generality the above conjecture is actually false, as we recently learned from M. Cowling [10, though it is still an open problem to determine under which minimal regularity conditions the smoothness assertion is true. In the present paper we assume smoothness and we prove finite dimensionality of the automorphism algebra of a closely related structure. In other words, we are interested in splittings of the ground layer of the kind $\mathfrak{g}_{-1}=\mathfrak{a}_{1}+\mathfrak{a}_{2}$ (or, more generally, into $m \geq 2$ subspaces), and we study the local diffeomorphisms of $G$ whose differentials preserve each of the bundles obtained by left translating $\mathfrak{a}_{1}$ and $\mathfrak{a}_{2}$.

As for Korányi's conjecture, he considers the following setup. We are given smooth distributions $\mathcal{D}_{1}, \ldots, \mathcal{D}_{m}$ on a manifold $M$ and we assume that their sections satisfy a joint Hörmander condition; that is, the repeated brackets of vector fields belonging to the various distributions $\mathcal{D}_{j}$ span the full tangent space at all points. The conjecture is that the Lie algebra of those vector fields $V$ on $M$ for which $[V, X] \in \mathcal{D}_{j}$ for all $X \in \mathcal{D}_{j}$ and all $j$ is finite dimensional under suitable nondegeneracy. In Korányi's words: Perhaps the following condition would be sufficient: at each point $p$, for each $i, j$ we define the form $L_{i j}(\xi, \eta)$ on $\mathcal{D}_{i, p} \times \mathcal{D}_{j, p}$ by extending $\xi$ and $\eta$ to vector fields $X, Y$ belonging to $\mathcal{D}_{i}$, respectively $\mathcal{D}_{j}$, and then taking $[X, Y]_{p}$ modulo $\mathcal{D}_{i, p}+\mathcal{D}_{j, p}$. For every $\xi \neq 0$ in $\mathcal{D}_{i, p}$ there should exist $j$ and $\eta \in \mathcal{D}_{j, p}$ such that $L_{i j}(\xi, \eta) \neq 0$.

We prove that if the subbundles $\mathcal{D}_{i}=H_{i} G$ satisfy the condition (ii) of Definition 2.1 below, which is modeled after Korányi's, then the vector fields for which $[V, X] \in \mathcal{D}_{j}$ for all $X \in \mathcal{D}_{j}$ and all $j$ form a finite dimensional Lie algebra.

\section{Preliminaries And notation}

A nilpotent Lie algebra $\mathfrak{g}$ over $\mathbb{R}$ has an $s$-step stratification if it can be written as

$$
\mathfrak{g}=\sum_{i=-s}^{-1} \mathfrak{g}_{i},
$$

a direct sum of vector spaces such that

$$
\left[\mathfrak{g}_{-1}, \mathfrak{g}_{j}\right]=\mathfrak{g}_{j-1},
$$

for every $j \leq-1$. A Carnot group $G$ is a connected, simply connected nilpotent Lie group whose Lie algebra is stratified and equipped with an inner product such that $\mathfrak{g}_{i} \perp \mathfrak{g}_{j}, i \neq j$. We denote the algebra of vector fields on $G$ by $\mathfrak{X}(G)$. By left translation, the space $\mathfrak{g}$ gives rise to the tangent bundle $T G$ and the subspace $\mathfrak{g}_{-1}$ defines a subbundle $H G$ of $T G$ which is called a horizontal bundle or contact bundle. Equation (2.1) implies that the horizontal bundle generates at each point the whole tangent space to $G$. A diffeomorphism $\phi: \mathcal{A} \rightarrow \mathcal{B}$ between open sets of $G$ is called a contact mapping if the differential $\phi_{*}$ preserves the horizontal bundle. By composition, the space of global contact mappings is a possibly infinite dimensional Lie group. If it is finite dimensional, $G$ is classically said to be rigid; otherwise $G$ is nonrigid. The contact condition can be read at the Lie algebra level. A contact vector field $V$ on an open set of $G$ is a smooth vector field which generates a local one-parameter flow $\phi_{t}$ of contact mappings. If $\tilde{X}$ is the left invariant vector field 
corresponding to the vector $X \in \mathfrak{g}_{-1}$, we have

$$
\left.\frac{d}{d t}\left(\phi_{t}\right)_{*}(\tilde{X})\right|_{t=0}=-\mathcal{L}_{V}(\tilde{X})=[\tilde{X}, V]
$$

where $\mathcal{L}$ denotes the Lie derivative. Thus a smooth vector field $V$ is a contact vector field if and only if

$$
[V, \tilde{X}] \in H G \text { for every } \tilde{X} \in H G \text {; }
$$

that is, ad $V$ preserves the horizontal bundle. Clearly, if a Carnot group $G$ admits an infinite dimensional space of contact vector fields, then $G$ is nonrigid in the classical sense. We are rather interested in the generalised notion of rigidity that was essentially introduced in [3, 4].

Definition 2.1. We say that the stratified algebra $\mathfrak{g}$ carries a multicontact structure if there exist vector subspaces $\mathfrak{a}_{1}, \ldots, \mathfrak{a}_{m}, m \geq 2$, of the first stratum $\mathfrak{g}_{-1}$ that satisfy the following two properties:

(i) $\mathfrak{g}_{-1}=\mathfrak{a}_{1}+\cdots+\mathfrak{a}_{m}$, a vector space direct sum;

(ii) for every $1 \in \underline{m}$ and for every $X \in \mathfrak{a}_{i}$ there exists $j \in \underline{m}, j \neq i$, and $Y \in \mathfrak{a}_{j}$ such that $[X, \bar{Y}] \neq 0$.

Remarks. (a) A sufficient condition for the existence of relevant multicontact structures is that

$$
\mathfrak{z}(\mathfrak{g}) \cap \mathfrak{g}_{-1}=\{0\},
$$

where $\mathfrak{z}(\mathfrak{g})$ denotes the center of $\mathfrak{g}$. Indeed, assume that (2.3) holds. Then any basis $\left\{X_{1}, \ldots, X_{p}\right\}$ of $\mathfrak{g}_{-1}$ gives rise to a "finest possible" multicontact structure by putting $\mathfrak{a}_{i}=\operatorname{span}\left\{X_{i}\right\}$ for every $i \in p$. We shall refer to these structures as $p$-contact structures, where $p=\operatorname{dim} \mathfrak{g}_{-1}$. Secondly, for any such basis there always exists a bicontact structure adapted to it, that is, a splitting of $\mathfrak{g}_{-1}$ into the direct sum of two subspaces $\mathfrak{a}_{1}$ and $\mathfrak{a}_{2}$ that satisfy (ii), each of which is spanned by a subset of basis elements. We show this by induction on $p$. If $p=2$, (2.3) assures that $\mathfrak{a}_{1}=\operatorname{span}\left\{X_{1}\right\}$ and $\mathfrak{a}_{2}=\operatorname{span}\left\{X_{2}\right\}$ satisfy (ii). For a higher $p$, first observe that the set $\left\{X_{1}, \ldots, X_{p-1}\right\}$ generates a subalgebra of $\mathfrak{g}$. By the induction hypothesis, there exist subspaces $\mathfrak{a}_{1}$ and $\mathfrak{a}_{2}$ of $\operatorname{span}\left\{X_{1}, \ldots, X_{p-1}\right\}$ satisfying properties (i) and (ii). Now, (2.3) implies that $\left[X_{p}, \mathfrak{a}_{i}\right] \neq\{0\}$ for at least one $i \in \underline{2}$, say $i=1$, so that $\mathfrak{a}_{1}$ and $\mathfrak{a}_{2}+\mathbb{R} X_{p}$ satisfy (ii).

Conversely, if (2.3) is not true, then bicontact structures may fail to exist. Take the stratified Lie algebra $\mathfrak{g}=\mathfrak{g}_{-1}+\mathfrak{g}_{-2}$, where $\mathfrak{g}_{-1}=\operatorname{sp}\left\{X_{1}, X_{2}, X_{3}\right\}, \mathfrak{g}_{-2}=\operatorname{sp}\{Y\}$ and the only nonzero brackets among basis elements are

$$
\left[X_{1}, X_{2}\right]=\left[X_{1}, X_{3}\right]=Y \text {. }
$$

It is immediate that $X_{2}-X_{3} \in \mathfrak{z}(\mathfrak{g}) \cap \mathfrak{g}_{-1}$. If $\mathfrak{g}_{-1}=\mathfrak{a}_{1}+\mathfrak{a}_{2}$ were a bicontact structure, then it would be possible to say $\mathfrak{a}_{1}=\operatorname{span}\{A, B\}$ and $\mathfrak{a}_{2}=\operatorname{span}\{C\}$ with linearly independent $A, B$ and $C$. Write $X_{2}-X_{3}=a A+b B+c C$. Then

$$
0=[a A+b B+c C, C]=[a A+b B, C] .
$$

Hence, if $a A+b B \neq 0$, then (ii) does not hold, whereas if $a A+b B=0$, then $\mathfrak{a}_{2} \subseteq \mathfrak{z}(\mathfrak{g})$ and again (ii) fails.

(b) Condition (2.3) is not automatically satisfied by all multicontact structures; that is, it is not a consequence of (ii), as the following example shows. Consider

\footnotetext{
${ }^{1}$ Here and in the following, for any positive integer $m$ we write $\underline{m}=\{1,2, \ldots, m\}$.
} 
the stratified Lie algebra $\mathfrak{g}=\mathfrak{g}_{-1}+\mathfrak{g}_{-2}$, where $\mathfrak{g}_{-1}=\operatorname{sp}\left\{X_{1}, X_{2}, X_{3}, X_{4}\right\}, \mathfrak{g}_{-2}=$ $\operatorname{sp}\left\{Y_{1}, Y_{2}\right\}$ and the only nonzero brackets among basis elements are

$$
\begin{aligned}
& {\left[X_{1}, X_{2}\right]=\left[X_{1}, X_{3}\right]=Y_{1},} \\
& {\left[X_{2}, X_{4}\right]=\left[X_{3}, X_{4}\right]=Y_{2} .}
\end{aligned}
$$

It is immediate that $X_{2}-X_{3} \in \mathfrak{z}(\mathfrak{g}) \cap \mathfrak{g}_{-1}$. Nevertheless, both of the following decompositions of $\mathfrak{g}_{-1}$ satisfy (ii):

$$
\begin{aligned}
& \mathfrak{g}_{-1}=\operatorname{sp}\left\{X_{1}, X_{2}\right\}+\operatorname{sp}\left\{X_{3}, X_{4}\right\}, \\
& \mathfrak{g}_{-1}=\operatorname{sp}\left\{X_{1}\right\}+\operatorname{sp}\left\{X_{2}\right\}+\operatorname{sp}\left\{X_{3}\right\}+\operatorname{sp}\left\{X_{4}\right\} .
\end{aligned}
$$

Indeed, for any $X=\alpha X_{1}+\beta X_{2} \neq 0$ there exists $X^{\prime}=\gamma X_{3}+\delta X_{4} \neq 0$ for which $\left[X, X^{\prime}\right] \neq 0$, for if $\alpha \neq 0$, we can take $X^{\prime}=X_{3}$; and if $\beta \neq 0$, we can take $X^{\prime}=X_{4}$. Similarly, for any $X^{\prime}=\gamma X_{3}+\delta X_{4} \neq 0$ there exists $X=\alpha X_{1}+\beta X_{2} \neq 0$ for which $\left[X, X^{\prime}\right] \neq 0$. Hence, the first splitting satisfies (ii). The second satisfies it trivially.

Suppose that $\mathfrak{g}$ is a stratified Lie algebra that carries a multicontact structure, and let $G$ denote the corresponding connected and simply connected group. By left translation, each $\mathfrak{a}_{i}$ generates a subbundle of the tangent bundle of $G$, denoted $H_{i} G$. A diffeomorphism $\phi$ between open subsets of $G$ whose differential preserves $H_{i} G$ at each point is called a multicontact mapping. We look at the infinitesimal version of this notion, that is, at the Lie algebra level. We say that a vector field $V$ on $G$ is a multicontact vector field if $\left[V, H_{i} G\right] \subset H_{i} G$. The Lie algebra of multicontact vector fields is the Lie algebra of the (possibly infinite dimensional) Lie group of multicontact mappings. We say that $G$ is rigid relative to the multicontact structure if the Lie algebra of multicontact vector fields is finite dimensional. We prove that this is always the case in Theorem 4.1 .

The nondegeneracy condition (ii) in Definition 2.1 seems somehow necessary. Indeed, if we weaken it by asking the requirement only for some of the spaces $\mathfrak{a}_{i}$, we can easily find examples showing that rigidity fails. Consider the Lie algebra $\mathfrak{g}=\operatorname{span}\left\{X_{1}, X_{2}, X_{3}, Y_{1}, Y_{2}\right\}$, where $\left[X_{1}, X_{2}\right]=Y_{1}$ and $\left[X_{2}, X_{3}\right]=Y_{2}$ are the only nonzero brackets. If $\mathfrak{a}_{1}=\operatorname{span}\left\{X_{1}, X_{2}\right\}$ and $\mathfrak{a}_{2}=\operatorname{span}\left\{X_{3}\right\}$, then for every vector in $\mathfrak{a}_{2}$ there exists a vector in $\mathfrak{a}_{1}$ with which it does not commute, but the converse is false, so (ii) is not true. Given exponential coordinates on $G$, it is easy to verify that $V=f \tilde{Y}_{1}+\left(\tilde{X}_{2} f\right) \tilde{X}_{1}$ satisfies $\left[V, H_{i} G\right] \subset H_{i} G$ for every $i \in \underline{2}$ and for every smooth function $f=f\left(x_{2}\right)$.

The proof of our main contribution rests on the results proved by Tanaka in [12] in the context of his study of infinitesimal automorphisms of dynamical systems associated to graded Lie algebras. In the next section we review some of the basic constructs.

\section{TANAKA PROLONGATION}

Tanaka introduces the notion of prolongation of a stratified nilpotent Lie algebra relative to a subalgebra $\mathfrak{g}_{0}$ of $\operatorname{Der}(\mathfrak{g})$, the strata preserving derivations of $\mathfrak{g}$. It is a Lie algebra $\mathcal{T}\left(\mathfrak{g}, \mathfrak{g}_{0}\right)$ with the following properties:

(i) $\mathcal{T}\left(\mathfrak{g}, \mathfrak{g}_{0}\right)=\sum_{-s \leq i} \mathfrak{g}_{i}$ is a graded Lie algebra;

(ii) if $\left[u, \mathfrak{g}_{-1}\right]=0$ for some $u \in \mathfrak{g}_{k}, k \geq 0$, then $u=0$;

(iii) $\mathcal{T}\left(\mathfrak{g}, \mathfrak{g}_{0}\right)$ is maximal among the Lie algebras containing $\mathfrak{g}$ and satisfying (i) and (ii). 
The construction of $\mathcal{T}\left(\mathfrak{g}, \mathfrak{g}_{0}\right)$ is as follows. First, we define a bracket on $\mathfrak{g}+\mathfrak{g}_{0}$ (direct sum of vector spaces) by keeping the brackets on $\mathfrak{g}$ and $\mathfrak{g}_{0}$ and by setting $[D, X]=D(X)$ if $D \in \mathfrak{g}_{0}, X \in \mathfrak{g}$. Skew-symmetry is imposed, so that $\mathfrak{g}+\mathfrak{g}_{0}$ is a Lie algebra. Inductively, for $k>0, \mathfrak{g}_{k}$ is defined as the space of all homomorphisms $u$ : $\mathfrak{g} \rightarrow \mathfrak{g}+\mathfrak{g}_{0}+\cdots+\mathfrak{g}_{k-1}$ for which $u\left(\mathfrak{g}_{j}\right) \subset \mathfrak{g}_{j+k}$ and $[u,[X, Y]]=[[u, X], Y]+[X,[u, Y]]$ for every $X, Y \in \mathfrak{g}$, where by definition $[u, Z]=u(Z)$.

The bracket of two elements in the prolongation is defined in such a way that the Jacobi identity is satisfied, that is,

$$
[u, v](X)=[[u, v], X]=[[u, X], v]+[u,[v, X]], \quad X \in \mathfrak{g} .
$$

We say that $\mathcal{T}\left(\mathfrak{g}, \mathfrak{g}_{0}\right)$ is of finite type if $\operatorname{dim} \mathcal{T}\left(\mathfrak{g}, \mathfrak{g}_{0}\right)<\infty$. Now, given $\mathcal{T}\left(\mathfrak{g}, \mathfrak{g}_{0}\right)=$ $\sum_{j \geq-s} \mathfrak{g}_{j}$ we define

$$
\mathfrak{h}_{p}=\left\{u \in \mathfrak{g}_{p}: u[X, Y]=0 \text { for every } X, Y \in \mathfrak{g}\right\}, \quad p \geq 0 .
$$

The following result is the content of Corollary 1 in [12], p. 76 .

Theorem 3.1 (N. Tanaka). The prolongation $\mathcal{T}\left(\mathfrak{g}, \mathfrak{g}_{0}\right)=\sum_{-s \leq i} \mathfrak{g}_{i}$ is of infinite type if and only if $\mathfrak{h}_{p} \neq\{0\}$ for all $p \geq 0$.

\section{Rigidity}

The structure of the Lie algebra of contact vector fields or multicontact vector fields is described by Tanaka prolongations. Choose

$$
\mathfrak{g}_{0}=\left\{D \in \operatorname{Der}(\mathfrak{g}): D \mathfrak{a}_{i} \subset \mathfrak{a}_{i}, i \in \underline{m}\right\} .
$$

From [12] it follows that $\mathcal{T}\left(\mathfrak{g} ; \mathfrak{g}_{0}\right)$ is of finite type if and only if

$$
\mathcal{M}(\mathfrak{g})=\left\{V \in \mathfrak{X}(G):\left[V, H_{i} G\right] \subset H_{i} G, i \in \underline{m}\right\}
$$

has finite dimension. Moreover, $\mathcal{T}\left(\mathfrak{g} ; \mathfrak{g}_{0}\right)$ and $\mathcal{M}(\mathfrak{g})$ are isomorphic when they have finite dimension (cf. [12, §6] or [15, §2.3]).

By Theorem 3.1, in order to prove that the space of multicontact vector fields on $G$ has finite dimension, it is enough to show that $\mathfrak{h}_{p}=\{0\}$ for some $p \geq 0$. In fact, we will see that $\mathfrak{h}_{1}=\{0\}$.

Theorem 4.1. Every multicontact structure on a Carnot group is rigid.

Proof. Given $u \in \mathfrak{h}_{1}, u[X, Y]=u(X) Y-u(Y) X=0$ for every $X, Y \in \mathfrak{g}$, so that in particular $u\left(\mathfrak{a}_{s}\right) \mathfrak{a}_{t}=u\left(\mathfrak{a}_{t}\right) \mathfrak{a}_{s}$. From $\S 11.2$ in [12, it follows that if $v \in \mathfrak{h}_{p}$ and $X \in \mathfrak{g}_{-1}$, then $v(X) \in \mathfrak{h}_{p-1}$, for every $p \geq 1$. In particular, we have $u\left(\mathfrak{a}_{s}\right) \subset \mathfrak{h}_{0}$. Hence $u\left(\mathfrak{a}_{s}\right) \mathfrak{a}_{t}=\{0\}$ whenever $t \neq s$ because $u\left(\mathfrak{a}_{s}\right) \mathfrak{a}_{t}=u\left(\mathfrak{a}_{t}\right) \mathfrak{a}_{s} \subset \mathfrak{a}_{t} \cap \mathfrak{a}_{s}=\{0\}$.

Now fix $s \in \underline{m}$ and select bases $\left\{X_{1}, \ldots, X_{p}\right\}$ of $\mathfrak{a}_{s},\left\{Y_{1}, \ldots, Y_{q}\right\}$ of $\mathfrak{a}_{*}=\mathfrak{a}_{1}+\cdots+$ $\mathfrak{a}_{s-1}+\mathfrak{a}_{s+1}+\cdots+\mathfrak{a}_{m}$ and $\left\{Z_{1}, \ldots, Z_{r}\right\}$ of $\mathfrak{g}_{-2}$. Since $\mathfrak{h}_{0}$ consists of the derivations which vanish on the strata $\mathfrak{g}_{j}$ with $j \leq-2$ and preserve each $\mathfrak{a}_{i}$, hence $\mathfrak{a}_{s}$ and $\mathfrak{a}_{*}$, we can represent its elements as matrices in $\mathfrak{g l}\left(\mathfrak{g}_{-1}\right)$. More precisely, if $D \in \mathfrak{h}_{0}$, then relative to the chosen bases,

$$
D=\left[\begin{array}{cc}
A_{s} & 0 \\
0 & A_{*}
\end{array}\right] .
$$

Formally, $A_{s}=\pi_{s}(D)$ and $A_{*}=\pi_{*}(D)$, where $\pi_{s}: \mathfrak{h}_{0} \rightarrow \mathfrak{g l}\left(\mathfrak{a}_{s}\right)$ and $\pi_{*}: \mathfrak{h}_{0} \rightarrow \mathfrak{g l}\left(\mathfrak{a}_{*}\right)$ are the natural projections. The fact that $u\left(\mathfrak{a}_{s}\right) \mathfrak{a}_{t}=\{0\}$ for every $t \neq s$ can thus be written as $\pi_{*}\left(u\left(\mathfrak{a}_{s}\right)\right)=\{0\}$. 
Now, for $i \in \underline{p}$ and $j \in \underline{q}$,

$$
\begin{aligned}
0 & =D\left[X_{i}, Y_{j}\right] \\
& =\left[\sum_{k=1}^{p} a_{s}^{k i} X_{k}, Y_{j}\right]+\left[X_{i}, \sum_{\ell=1}^{q} a_{*}^{\ell j} Y_{\ell}\right] \\
& =\sum_{\mu=1}^{n} \sum_{k=1}^{p} a_{s}^{k i} \alpha_{k j}^{\mu} Z_{\mu}+\sum_{\nu=1}^{n} \sum_{\ell=1}^{q} a_{*}^{\ell j} \alpha_{i \ell}^{\nu} Z_{\nu},
\end{aligned}
$$

where $\alpha_{i j}^{\mu}$ are the structure constants defined by $\left[X_{i}, Y_{j}\right]=\sum_{\mu=1}^{n} \alpha_{i j}^{\mu} Z_{\mu}$. Therefore, for every $i \in \underline{p}, j \in \underline{q}$ and $\mu \in \underline{n}$,

$$
\sum_{k=1}^{p} a_{s}^{k i} \alpha_{k j}^{\mu}+\sum_{\ell=1}^{q} a_{*}^{\ell j} \alpha_{i \ell}^{\mu}=0 .
$$

Now put $V_{s}=\pi_{s}\left(\mathfrak{h}_{0}\right)$ and $V_{*}=\pi_{*}\left(\mathfrak{h}_{0}\right)$, and define

$$
\begin{array}{rlrl}
\Phi_{s}: V_{s} & \rightarrow \mathbb{R}^{p q r}, & A_{s} & \mapsto \sum_{i, j, \mu}\left(\sum_{k} a_{s}^{k i} \alpha_{k j}^{\mu}\right) e_{i j \mu}, \\
\Phi_{*}: V_{*} \rightarrow \mathbb{R}^{p q r}, & A_{*} \mapsto \sum_{i, j, \mu}\left(\sum_{\ell} a_{*}^{\ell j} \alpha_{i \ell}^{\mu}\right) e_{i j \mu} .
\end{array}
$$

Then (4.1) reads

$$
\Phi_{s}\left(A_{s}\right)+\Phi_{*}\left(A_{*}\right)=0,
$$

which implies in particular that $\Phi_{s}$ and $\Phi_{*}$ have the same image, say $W$. We show next that $\Phi_{s}$ is injective. Indeed, let $A_{s}=\pi_{s} D$ be such that $\Phi_{s}\left(A_{s}\right)=0$. Since for every $i \in \underline{p}$ and $j \in \underline{q}$,

$$
\left[\sum_{k=1}^{p} a_{s}^{k i} X_{k}, Y_{j}\right]=\sum_{\mu=1}^{n} \sum_{k=1}^{p} a_{s}^{k i} \alpha_{k j}^{\mu} Z_{\mu}=\sum_{\mu=1}^{n}\left(\Phi_{s}\left(A_{s}\right)\right)_{i j \mu} Z_{\mu}=0,
$$

it follows that $\left[X^{(i)}, \mathfrak{a}_{*}\right]=0$ for every $i \in \underline{p}$, where $X^{(i)}=\sum_{k=1}^{p} a_{s}^{k i} X_{k} \in \mathfrak{a}_{s}$. The nondegeneracy condition (ii) in Definition 2.1 implies that $X^{(i)}=0$ for every $i \in \underline{p}$, hence that $A_{s}=0$, thereby showing injectivity. If $\tilde{\Phi}_{s}: V_{s} \rightarrow W$ denotes the bijective linear mapping defined by $\Phi_{s}$, equation (4.2) gives

$$
\pi_{s}(D)=A_{s}=-\tilde{\Phi}_{s}^{-1} \circ \Phi_{*}\left(A_{*}\right)=-\tilde{\Phi}_{s}^{-1} \circ \Phi_{*} \circ \pi_{*}(D)
$$

for every $D \in \mathfrak{h}_{0}$, that is, $\pi_{s}=-\tilde{\Phi}_{s}^{-1} \circ \Phi_{*} \circ \pi_{*}$. It follows that

$$
u\left(\mathfrak{a}_{s}\right)=\pi_{s}\left(u\left(\mathfrak{a}_{s}\right)\right)=-\tilde{\Phi}_{s}^{-1} \circ \Phi_{*} \circ \pi_{*}\left(u\left(\mathfrak{a}_{s}\right)\right)=\{0\}
$$

because, as observed earlier, $\pi_{*}\left(u\left(\mathfrak{a}_{s}\right)\right)=\{0\}$. Therefore $u\left(\mathfrak{g}_{-1}\right)=\{0\}$ and so $u=0$, as desired.

\section{REFERENCES}

1. A. Čap, M. Cowling, F. De Mari, M. Eastwood, R. McCallum, The Heisenberg group, SL(3, $\mathbb{R})$, and rigidity. Lect. Notes Ser. Inst. Math. Sci. Natl. Univ. Singap., 12, World Sci. Publ., Hackensack, NJ, 2007. MR2401809 (2009g:22015)

2. L. Capogna, M. Cowling, Conformality and Q-harmonicity in Carnot groups. Duke Math. J. 135 (2006), no. 3, 455-479. MR2272973(2007h:30017) 
3. M. Cowling, F. De Mari, A. Korányi, H.M. Reimann, Contact and conformal maps on Iwasawa $N$ groups. Atti Accad. Naz. Lincei Cl. Sci. Fis. Mat. Natur. Rend. Lincei (9) Mat. Appl. 13 (2002), 219-232. MR.1984102 (2004f:53028)

4. M. Cowling, F. De Mari, A. Korányi, H.M. Reimann, Contact and conformal maps in parabolic geometry. I, Geom. Dedicata 111 (2005), 65-86. MR2155176 (2006d:53049)

5. L. Hörmander, Hypoelliptic second order differential equations. Acta Math. 119 (1967), 147171. MR0222474 (36:5526)

6. A. Korányi, Multicontact maps: Results and conjectures. Lect. Notes Semin. Interdiscip. Mat., IV, S.I.M. Dep. Mat. Univ. Basilicata, Potenza, 2005, 57-63. MR2222536 (2006m:53123)

7. A. Korányi, H.M. Reimann, Quasiconformal mappings on the Heisenberg group. Invent. Math. 80 (1985), no. 2, 309-338. MR788413 (86m:32035)

8. A. Ottazzi, Multicontact vector fields on Hessenberg manifolds. J. Lie Theory 15 (2005), no. 2, 357-377. MR2147433 (2006c:22015)

9. A. Ottazzi, A sufficient condition for nonrigidity of Carnot groups. Math. Z. 259 (2008), no. 3, 617-629. MR2395129 (2009b:22007)

10. M. Cowling, Mappings of groups that send cosets to cosets. Communication at the workshop "Geometric and harmonic analysis on subriemannian spaces", Milan, 16-18 March, 2009.

11. S. Sternberg. Lectures on differential geometry. Prentice-Hall, Englewood Cliffs, NJ, 1964. MR0193578 (33:1797)

12. N. Tanaka, On differential systems, graded Lie algebras and pseudogroups. J. Math. Kyoto Univ. 10 (1970), 1-82. MR0266258 (42:1165)

13. N. Tanaka, On affine symmetric spaces and the automorphism groups of product manifolds. Hokkaido Math. J. 14 (1985), no. 3, 277-351. MR808817 (87c:53100)

14. I. Venturi, Rigid 2-step nilpotent groups relative to multicontact structures, to appear in Rend. Semin. Mat. Univ. Padova.

15. K. Yamaguchi, Differential systems associated with simple graded Lie algebras. Progress in differential geometry, vol. 22 of Adv. Stud. Pure Math., pages 413-494. Math. Soc. Japan, Tokyo, 1993. MR1274961 (95g:58263)

16. T. Yatsui, On pseudo-product graded Lie algebras. Hokkaido Math. J. 17 (1988), no. 3, 333343. MR965191 (90b:17037)

Dipartimento di Matematica, Università di Genova, Genova, Italy

E-mail address: demari@dima.unige.it

Dipartimento di Matematica e Applicazioni, Università di Milano "Bicocca", via Cozzi, 53, 20125 Milano, Italy

E-mail address: alessandro.ottazzi@unimib.it 